\title{
Telepsychiatry for stable Chinese psychiatric out- patients in custody in Hong Kong: a case-control pilot study
}

\author{
KM Cheng, Bonnie WM Siu *, Cherie CY Au Yeung, TP Chiang, MH So, Mick CW Yeung
}

\section{A B S T R A C T}

Introduction: In Hong Kong, persons in custody receive primary medical care within the institutions of the Correctional Services Department (CSD). However, for psychiatric care, persons in custody must attend specialist out-patient clinics (SOPCs), which may cause embarrassment and stigmatisation. The aim of this interventional pilot study was to compare teleconsultations with faceto-face consultations for a group of stable Chinese psychiatric out-patients in custody.

Methods: A total of 86 stable Chinese male outpatients in custody were recruited for psychiatric teleconsultations. They were compared with 249 age-matched Chinese male out-patients in custody attending standard face-to-face psychiatric consultations at other SOPCs. The two groups had comparable baseline characteristics including age, education level, and 12-item Chinese General Health Questionnaire (C-GHQ-12) score. A satisfaction survey of patients towards the teleconsultation was also carried out.
Results: Compared with the face-to-face consultation group, the teleconsultation group showed a significantly better result in the difference in C-GHQ-12 scores before and after consultations $(\mathrm{P}=0.023)$. The correlation between the first and second teleconsultations also showed a moderate positive relationship $(r=0.309)$. The satisfaction survey showed a favourable response to teleconsultations. No significant adverse events were identified for the teleconsultation group.

Conclusions: The results suggest that teleconsultations are a sustainable and safe alternative to face-to-face consultations for stable Chinese psychiatric out-patients in custody.
Hong Kong Med J 2018;24:378-83 DOI: 10.12809/hkmj187217

\begin{abstract}
${ }^{1}$ KM Cheng, MB, BS, FHKAM (Psychiatry)
\end{abstract}
'BWM Siu *, MB, ChB, FHKAM (Psychiatry)
${ }^{2}$ CCY Au Yeung, BSc, MStat
${ }^{1}$ TP Chiang, MB, BS, FHKAM (Psychiatry)
${ }^{3}$ MH So, BSc
${ }^{3}$ MCW Yeung, FHKAN, BSC
Department of General Adult Psychiatry, Castle Peak Hospital, Tuen Mun, Hong Kong Hong Kong
2 Department of Forensic Psychiatry, Castle Peak Hospital, Tuen Mun,
${ }^{3}$ Correctional Services Department, Wanchai, Hong Kong
* Corresponding author: swm810@ha.org.hk

\section{New knowledge added by this study \\ Psychiatric teleconsultations are a sustainable and safe alternative to face-to-face consultations for stable Chinese psychiatric out-patients in custody. \\ Implications for clinical practice or policy \\ - The intrinsic problems of embarrassment and stigmatisation caused to persons in custody, their risk of abscondence, and the issue of general public safety can all be addressed with this promising alternative mode of psychiatric care for stable Chinese psychiatric out-patients in custody.}

\section{Introduction}

Telepsychiatry is the practice of delivering mental health consultations at a distance. New developments in information and communication technologies have allowed telepsychiatry to become a viable method of providing services to patients in rural or remote locations with limited access to medical services..$^{1-3}$ Telepsychiatry has been used in prison settings for more than 20 years. ${ }^{4} \mathrm{~A}$ demonstration of telepsychiatry in prison in the US in 1996 concluded that this practice was cost-effective. ${ }^{5}$ Prison administrators even claimed that there were fewer assault incidents after its use. ${ }^{5}$ In Hong Kong, the use of telepsychiatry can be dated back to $1998 .{ }^{6}$

Currently, persons in custody (PICs) in Hong Kong receive primary medical care within the institutions of the Correctional Services Department (CSD). However, for specialist psychiatric service for their mental problems, PICs must attend psychiatric specialist out-patient clinics (SOPCs) of the Hospital Authority. In addition, most psychiatric drugs are available only in SOPCs.

For security reasons, PICs must be escorted by at least two CSD staff and be handcuffed on every occasion they need to attend follow-up at a SOPC. Such an exposing arrangement inevitably 
causes much embarrassment and stigmatisation for PICs. There is also a potential risk to the public in the event of abscondence from custody. The PIC may also experience travel sickness during the journey between the correctional facilities and the SOPC; most correctional facilities in Hong Kong are situated in relatively remote areas, so the journey times can be long. Furthermore, other patients in the SOPC may feel uncomfortable witnessing a PIC being handcuffed. Some SOPCs manage this problem by placing the PIC in a special corner or room, depending on availability.

Face-to-face consultation is the gold standard for medical practice. However, telepsychiatry is suitable for PICs and might confer additional benefits for this group of patients. Direct physical examinations are typically unnecessary for stable psychiatric patients during follow-up. Additionally, the nurse at the CSD site can help measure vital signs such as blood pressure, pulse, and temperature. Therefore, offering PICs psychiatric teleconsultations cannot only maintain their usual psychiatric care but also reduce embarrassment, stigmatisation, and the risk of abscondence. Furthermore, it can also reduce the need for special arrangements in SOPCs.

To the best of our knowledge, there have been no previous studies exploring the effect of psychiatric teleconsultations for Chinese psychiatric out-patients under the legal custody of the CSD in Hong Kong. The main aim of the present study was to explore the use of psychiatric teleconsultations for stable Chinese psychiatric out-patients under the legal custody of the CSD in Hong Kong. The desired outcome was to maintain the clinical interests of PICs and to provide them with appropriate psychiatric services using telecommunications in a safe, humane, and cost-effective manner. This was an interventional pilot study evaluating the effect of psychiatric teleconsultations on the general health of an intervention group of clinically stable Chinese male psychiatric out-patients who were under the custody of the CSD as compared with a matched control group of Chinese male psychiatric out-patients under the usual type of care (ie, faceto-face consultation with a psychiatrist at a SOPC). In addition, the satisfaction of patients towards psychiatric teleconsultations was assessed.

The null hypotheses of this study were as follows:

1. After the consultation, the psychological health of the intervention group is worse than that of the control group;

2. The effect of psychiatric teleconsultations is unsustainable;

3. Patients in the intervention group are unsatisfied with the psychiatric teleconsultations;

4. Adverse events occur during psychiatric teleconsultations.

\section{視像遙距診症於精神穩定的在囚精神科病人的應 用：病例對照試點研究 \\ 鄭蓋民、蕭慧敏、歐陽卓儀、蔣天寶、蘇文軒、楊俊偉}

引言：香港懲教署可為在囚人士提供基本醫療服務。然而, 在囚人士 若須往精神科專科診所接受治療或會感到壏伧和被歧視。這項干預試 點研究旨在比較精神穩定的在囚精神科患者於精神科專科診所接受視 像遙距診症和面談診症的分別。

方法：86位精神穩定的在囚華籍精神科男性患者（干預組）獲邀在香 港一間精神科專科診所接受視像遙距診症, 另249名年齡相若而在其 他精神科專科診所接受傳統面談方式門診的在囚華籍精神科男性患者 被選為對照組。進行研究前兩組患者在年齡、教育程度以及 12 項一般 健康問卷的中文版本所得分數均沒有差異。研究團隊為病人對視像遙 距診症的滿意程度進行問卷調查。

結果：與對照組比較, 干預組覆診後於 12 項一般健康中文版問卷所得 的分數有較佳改善 $(\mathrm{P}=0.023)$ 。首次與第二次視像遙距診症的分數 亦顯示中等正相關（ $r=0.309 ） 。 干$ 預組中沒有發生不良事件, 患者 對視像遙距診症也表示滿意。

結論：視像遙距診症對於精神穩定的在囚華籍精神科患者是一個可持 續和安全的選擇。

\section{Methods}

This was an interventional pilot study conducted by the Hospital Authority in collaboration with the Hong Kong CSD.

\section{Participants}

The study period was from June 2014 to May 2016. Participants were aged 21 to 64 years. The intervention group comprised Chinese patients in custody attending follow-up at the SOPC of Castle Peak Hospital ( $\mathrm{CPH})$, Hong Kong. The control group included Chinese patients in custody attending follow-up at other SOPCs in Hong Kong. In this study, only male PICs were included because of logistic and feasibility reasons. Exclusion criteria applied when selecting intervention and control participants for this study included: (a) patients with mental instability or with prominent and recent change/ deterioration in mental condition, such as those who were suicidal or homicidal, or who had delirium or acute psychosis; (b) patients who required regular blood tests, such as those taking clozapine; (c) patients requiring other tests/investigations only available in SOPC or Hospital Authority hospitals; (d) patients requiring drug administration in SOPC, such as depot antipsychotics; (e) patients attending SOPC for the first time; or (f) patients with visual or auditory deficits that might impair the ability to interact via video-conferencing. Eligible patients meeting the inclusion criteria were invited to participate in the study. Written informed consent was obtained from the intervention and control participants. 


\section{Sample size}

A sample size of at least 80 participants for the intervention group with an intervention-to-control ratio of approximately 1:3 was adopted in this study. This was an affordable and representative sample size with reference to the number of stable psychiatric out-patients in custody attending followup appointment at the SOPC of $\mathrm{CPH}$ during the study period.

\section{Assessment tools}

Socio-demographic and clinical data including age, education level, and principal psychiatric diagnosis according to the 10th revision of the International Classification of Diseases were obtained. ${ }^{7}$ The intervention and control participants were requested to complete the Chinese version of the 12-item General Health Questionnaire (C-GHQ-12). ${ }^{8}$ The GHQ is a self-administered test used for evaluating the psychological components of ill health and is helpful in screening for general emotional distress. ${ }^{9,10}$ The GHQ possesses adequate content validity and construct validity, and good internal consistency has been demonstrated with Cronbach's alphas ranging from 0.82 to $0.93 .{ }^{9,10}$ The Chinese and English versions of the GHQ have been adopted for Chinese and nonChinese subjects, respectively. ${ }^{8-10}$ The C-GHQ-12 consists of 12 items, with each item assessing the severity of a mental problem using a 4-point Likert scale. $^{8}$ The six positive items were rated from 1 (more than usual) to 4 (much less than usual) and the six negative items from 1 (never) to 4 (much more than usual); thus, a higher score indicates a more severe mental health condition. In this study, the pre-consultation and post-consultation C-GHQ-12 scores for each patient were obtained. The difference between the two scores (ie, the pre-post difference) was used as a proxy measurement of the quality of consultation.

The intervention participants were also requested to complete a questionnaire in Chinese

TABLE I. Statements and questions in the satisfaction survey questionnaire (English translation)

1. The staff explained the psychiatric teleconsultation to me clearly and provided enough information on the psychiatric teleconsultation to me.

2. The telepsychiatry staff respected me and protected my privacy.

3. Were you satisfied with the audio quality of the telepsychiatry equipment?

4. Were you satisfied with the video quality of the telepsychiatry equipment?

5. The psychiatric teleconsultation met my medical care needs (eg, I was able to talk freely. The psychiatrist listened to my concerns.)

6. I felt less embarrassed in the psychiatric teleconsultation than in a face-toface consultation at the clinic.

7. I would like to use telepsychiatry for my next follow-up consultation session.

8. I would like to recommend psychiatric teleconsultations to others.

9. How was your overall psychiatric teleconsultation experience? designed to measurethe patient satisfaction regarding the psychiatric teleconsultation. The questionnaire consisted of nine statements/questions rated according to a 5-point Likert scale, from 1 (strongly agree) to 5 (strongly disagree) or from 1 (very satisfied) to 5 (very dissatisfied). The questionnaire was designed by the authors as there were no available validated Chinese questionnaires suitable for assessing patient satisfaction of telepsychiatry at the time of the study (English translations of the statements/questions in the questionnaire are listed in Table 1).

\section{Procedure}

The intervention participants were transferred from various CSD institutions to the Lai Chi Kok Reception Centre, Hong Kong, for the psychiatric teleconsultation. On the scheduled day of consultation, the CSD staff brought two portable video-conferencing devices to $\mathrm{CPH}$. Registration was performed only after the device had been checked as functional. All persons in the consultation rooms at the CSD site and at the $\mathrm{CPH}$ site were identified to each other prior to the consultation session. Consultation rooms provided at both sites were appropriately set up with particular attention to audio and visual privacy, lighting, backdrop, and gaze angle. A qualified CSD nurse was present in the consultation room at the CSD site together with the patient. There was also a CSD medical doctor at the reception centre during the psychiatric teleconsultation, in case emergency medical treatment was needed. After the consultation, the CSD staff collected the medicine for the patient according to the usual procedures. For the intervention participants, the maximum number of consecutive psychiatric teleconsultations was set as four, after which a face-to-face follow-up consultation must follow. The control participants attended only face-to-face consultations at other SOPCs. Both groups of participants filled in the C-GHQ-12 within 7 days before the consultation and again within 7 days after the consultation. In addition, the intervention participants filled in the satisfaction survey questionnaire after the psychiatric teleconsultation. Any major adverse events, such as medical or psychiatric emergencies, were recorded.

\section{Statistical analysis}

Descriptive statistics were used to analyse the baseline profile of the participants' sociodemographic and clinical characteristics as well as pre- and post-consultation C-GHQ-12 scores and satisfaction survey questionnaire responses. Chi squared test and two-samples $t$ test were performed to assess if there were differences in the baseline characteristics between the intervention and control participants. A non-parametric Mann-Whitney $U$ 
test was performed to test if there were differences in the pre-post difference in C-GHQ-12 score between intervention and control participants attending their first consultation. Spearman's correlation was used to compute the correlation between the prepost difference in C-GHQ-12 score of the first and second teleconsultations among the intervention participants. All statistical analyses were conducted using SPSS for Windows, version 12.0 (SPSS Inc, Chicago [IL], US), with $\mathrm{P}<0.05$ considered as statistically significant in this study.

\section{Results}

During the study period, there were 377 PIC scheduled attendances at CPH. Of these, 221 PIC scheduled attendances were suitable for psychiatric teleconsultation; however, for 49 of the suitable PIC scheduled attendances, the PICs refused to give consent for this study. Finally, 172 PIC psychiatric teleconsultation attendances were included. Each participant could have more than one psychiatric teleconsultation attendance during the study period. Therefore, 86 participants aged 21 to 64 years who were stable Chinese male psychiatric out-patients and who fulfilled the inclusion and exclusion criteria for psychiatric teleconsultations in $\mathrm{CPH}$ were included. For the control group, 249 male patients within the same age range (21-64 years) were recruited.

Table 2 compares patient characteristics between the intervention and control groups. There were no significant differences in the age and education profile between the two groups. The mean age of both groups was approximately 40 years.
Approximately three quarters of the participants in each group had attained education at the secondary level or above. There was a significant difference in the principal psychiatric diagnosis $(\mathrm{P}=0.029)$. Slightly over $50 \%$ of each group were diagnosed as substance abuse. A larger proportion of intervention participants had schizophrenia (28\%) than did the control participants (16\%). There were no significant differences in the mean pre-consultation C-GHQ-12 score between the two groups.

The mean (standard deviation) on-air time duration of the psychiatric teleconsultations was 6.33 (3.58) minutes. There were no significant adverse events associated with teleconsultations reported during the study. The pre-post difference in C-GHQ-12 score of the intervention participants was significantly higher than that of the control participants $(\mathrm{P}=0.023$; Table 2). Furthermore, among 29 intervention participants who had at least two teleconsultations, the association between prepost difference in C-GHQ-12 score of the first and second teleconsultations was moderately strong ( $r=0.309, \mathrm{P}=0.103$ ) but did not reach the level of significance set for this study. The possible scores on the satisfaction survey questionnaire ranged from 9 (the most satisfied) to 45 (the least satisfied). The mean (standard deviation) satisfaction score of the intervention group was 16.48 (4.35). No major adverse events were reported throughout the study.

\section{Discussion}

Telepsychiatry is not a new development in Hong Kong. Since 2001, the use of telepsychiatry has been shown to increase access to care. ${ }^{11}$ Studies have shown

TABLE 2. Demographic and clinical characteristics of participants in the intervention and control groups*

\begin{tabular}{|c|c|c|c|}
\hline & $\begin{array}{l}\text { Intervention group } \\
(\mathrm{n}=86)\end{array}$ & $\begin{array}{l}\text { Control group } \\
(n=249)\end{array}$ & $P$ value \\
\hline Age (years) & $40.4 \pm 11.0$ & $40.2 \pm 9.2$ & 0.922 \\
\hline Education & & & 0.410 \\
\hline Primary & $22(25.6)$ & $53(21.3)$ & \\
\hline Secondary or above & $64(74.4)$ & $196(78.7)$ & \\
\hline Principal diagnosis & & & 0.029 \\
\hline Substance abuse & $45(52.3)$ & $129(51.8)$ & \\
\hline Schizophrenia & $24(27.9)$ & $39(15.7)$ & \\
\hline Affective disorder & $5(5.8)$ & $18(7.2)$ & \\
\hline Others & $12(14.0)$ & $63(25.3)$ & \\
\hline Pre-consultation C-GHQ-12 score & $25.5 \pm 5.6$ & $25.6 \pm 6.3$ & 0.894 \\
\hline Median (IQR) & $24.0(24-28)$ & $24.0(22-29)$ & \\
\hline Post-consultation C-GHQ-12 score & $22.5 \pm 5.3$ & $23.5 \pm 5.5$ & 0.154 \\
\hline Median (IQR) & $22.0(20-24)$ & $24(20-26)$ & \\
\hline $\begin{array}{l}\text { Difference between pre- and post-consultation C-GHQ-12 } \\
\text { score for first attendance }\end{array}$ & $3.03 \pm 5.9$ & $2.25 \pm 5.4$ & 0.023 \\
\hline Median (IQR) & $3(0-6)$ & $2(-2.5$ to 2$)$ & \\
\hline
\end{tabular}

Abbreviations: C-GHQ-12 = 12-item Chinese General Health Questionnaire; IQR = interquartile range

* Date are shown as mean \pm standard deviation or No. (\%) of subjects, unless otherwise specified 
that telepsychiatry is an effective means to provide psychogeriatric services to residents of care homes, ${ }^{11}$ and cognitive intervention for community-dwelling elderly patients with memory problems. ${ }^{12}$ This was the first intervention pilot study in Hong Kong exploring the effect of psychiatric teleconsultation for Chinese psychiatric out-patients under the legal custody of the CSD. Our study compared the effectiveness of psychiatric teleconsultations with that of face-to-face consultations among PIC receiving out-patient psychiatric treatment. The two groups of stable Chinese male out-patient participants had the same baseline characteristics in age, education level, and pre-consultation C-GHQ12 score. The results showed that the standard of care of teleconsultations was comparable to that of face-to-face consultations. The pre-post difference in C-GHQ-12 score for teleconsultations had a marginally larger positive increase than did faceto-face consultations. The intervention participants also showed high satisfaction with the psychiatric teleconsultation service, with a mean satisfaction score above the 80th percentile. This results is similar to that of a previous study in the US that compared the effectiveness of telepsychiatry and in-person psychiatry sessions among 71 parolees over a 6-month follow-up period, revealing high satisfaction with telepsychiatry treatment. ${ }^{13}$ In the present study, the pre-post difference between CGHQ-12 score of the first and second psychiatric teleconsultations showed a moderate positive relationship, suggesting a consistent and sustainable clinical effect of telepsychiatry between sessions. Importantly, there were no reports of significant adverse events. Therefore, telepsychiatry can be considered sustainable and safe for Chinese PIC in Hong Kong.

Studies on the effects of telepsychiatry for incarcerated populations are relatively scarce; however, the results of our study are also consistent with studies of telepsychiatry in populations that were not involved with the correctional system. In a randomised controlled study in Canada, 495 patients were assigned at random to be examined face-toface or by telepsychiatry. ${ }^{14}$ Psychiatric consultations and follow-ups delivered by telepsychiatry produced clinical outcomes equivalent to those achieved when the service was provided face-to-face. ${ }^{14}$ This result suggests that psychiatric consultation and short-term follow-up can be as effective when delivered by telepsychiatry as when provided faceto-face. Another study evaluating the effectiveness of telepsychiatry in relation to cognitive changes in patients with dementia revealed that changes in the Mini-Mental State Examination score were not significantly different between patients receiving teleconsultations and those receiving clinic-based face-to-face consultations. ${ }^{15}$ This finding suggest that telepsychiatry may be a useful alternative to face-to- face clinical visits for treatment of a wide range of patient groups, including patients with dementia. Research has shown that there is an association between dementia and criminal offences and that the use of telepsychiatry might be extended to PIC with dementia in Hong Kong. ${ }^{16}$

Our study has several limitations. First, the sample size of the intervention group was small. Most enrolled patients served short sentences but had long follow-up intervals, because we recruited stable patients. Although the study duration was 2 years, only 29 out of 86 intervention participants had at least two psychiatric teleconsultations for comparison within the intervention group. Second, recruitment of potential telepsychiatry participants was limited to stable Chinese male psychiatric outpatients. Therefore, the sample may be affected by self-selection bias, because PICs volunteered to participate in telepsychiatry. This restricted sample also limits the generalisability of the results. Third, the mean time between the consultation and completion of the C-GHQ-12 was not recorded for intervention or control groups. Differences in this time interval may affect the pre-post difference in C-GHQ-12 score for the groups. We also did not collect data on the follow-up interval between the first and second psychiatric teleconsultations for the intervention group. This follow-up interval may affect the C-GHQ-12 score and the satisfaction on psychiatric teleconsultation. Fourth, the mean on-air time duration of psychiatric teleconsultations was 6.33 minutes; however, we did not measure the duration of face-to-face consultations for comparison between the two groups. The duration of consultation may have an effect on the outcome scores and patient satisfaction. Last, the study lacked robust clinical outcome measures and the satisfactory questionnaire adopted in this study had not been validated. Despite the limitations of this study, the results suggest that psychiatric teleconsultations are a sustainable and safe alternative to face-to-face consultations for stable Chinese male psychiatric out-patients in custody. The use of psychiatric teleconsultations has potential in other populations of PICs, such as female PICs or elderly PICs, but further research is required to investigate psychiatric teleconsultations for these populations.

Further research is required to examine the full potential of telepsychiatry among PICs in Hong Kong. In future studies, female patients should be recruited, to assess any sex-based differences. In addition, the scale of future studies using telepsychiatry can be increased by setting up more stations at CSD institutions and at other SOPCs in the Hospital Authority. Clinical outcomes such as symptom severity and psychological functioning of the patients could be assessed. Given the increasing number of older PICs in Hong Kong, recruitment of older patients could be considered for further study. 
It would be worthwhile to perform a future study with a larger sample size and with participants receiving a greater number of psychiatric teleconsultations, in order to further support the sustainability of psychiatric teleconsultations.

A cost analysis for psychiatric teleconsultation was beyond the scope of the present study. However, a systematic review of 137 telemedicine services in hospital facilities revealed that one of the key reasons for introducing telemedicine was cost reduction. ${ }^{17}$ Similar cost-saving conclusions have been reported in two studies in Hong Kong related to dementia and community geriatric services. ${ }^{11,12}$ In future studies, cost analysis of psychiatric teleconsultation including direct, indirect, and hidden costs could be calculated for further exploring the effectiveness of psychiatric teleconsultation.

\section{Conclusions}

Telepsychiatry appears to be an acceptable approach for providing out-patient psychiatric care for stable Chinese male PICs in Hong Kong. Telepsychiatry can be considered a sustainable and safe alternative to face-to-face consultations, with a comparable standard of care. Moreover, the intrinsic problems of embarrassment and stigmatisation caused to PICs, the risk that PICs might abscond, and the safety of the general public are all addressed by this promising alternative mode of psychiatric care for stable Chinese male PICs. Telepsychiatry is likely to show similar benefits for Chinese female PICs and PICs in other age-groups, such as older adults, but further research is required to confirm this.

\section{Author contributions}

All authors have made substantial contributions to the concept and design, acquisition of data, analysis and interpretation of data, drafting and critical revision for important intellectual content of the article.

\section{Acknowledgement}

We would like to acknowledge Dr CK Tung, Dr CF Tsui, Mr KW Chung, Mr Kenny Wong, Dr NM Kwong, and all the mental health professionals and CSD staff who have assisted in the design and implementation of this study. We would also like to acknowledge the study participants who had kindly participated in the present study.

\section{Funding/support}

This research received no specific grant from any funding agency in the public, commercial, or not-for-profit sectors.

\section{Declaration}

All authors have disclosed no conflicts of interest. All authors had full access to the data, contributed to the study, approved the final version for publication, and take responsibility for its accuracy and integrity. The research was presented in part in the Hospital Authority Convention 2017, 16 May 2017, Hong Kong.

\section{Ethical approval}

Approval for conducting the study was granted by the Research and Ethics Committee of the New Territories West Cluster of the Hospital Authority and the Research and Ethics Committee of the Correctional Services Department. The principles outlined in the Declaration of Helsinki were followed in the conduction of this study.

\section{References}

1. Gray LC, Edirippulige S, Smith AC, et al. Telehealth for nursing homes: the utilization of specialist services for residential care. J Telemed Telecare 2012;18:142-6.

2. Grady B, Myers KM, Nelson EL, et al. Evidence-based practice for telemental health. Telemed e-Health 2011;17:131-48.

3. WHO Global Observatory for eHealth. 2010. Telemedicine: opportunities and developments in Member States: report on the second global survey on eHealth. Geneva: World Health Organization. Available from: http://www.who.int/iris/handle/10665/44497. Accessed 1 Jan 2018.

4. McLaren P. Telemedicine and telecare: what can it offer mental health services? Adv Psychiatr Treat 2003;9:54-61.

5. Telemedicine can Reduce Correctional Health Care Costs: An Evaluation of a Prison Telemedicine Network. US Department of Justice; 1999.

6. Hjelm NM. Telemedicine: academic and professional aspects. Hong Kong Med J 1998;4:289-92.

7. International Statistical Classification of Diseases and Related Health Problems, 10th revision. Geneva, World Health Organization; 1992.

8. Chan DW. The Chinese version of the General Health Questionnaire: does language make a difference? Psychol Med 1985;15:147-55.

9. Goldberg DP. The Detection of Psychiatric Illness by Questionnaire. London: Oxford University Press; 1972.

10. Goldberg D, Williams P. A User's Guide to the General Health Questionnaire. London: NFER; 1988.

11. Tang WK, Chiu H, Woo J, Hjelm M, Hui E. Telepsychiatry in psychogeriatric service: a pilot study. Int J Geriatr Psychiatry 2001;16:88-93.

12. Poon P, Hui E, Dai D, Kwok T, Woo J. Cognitive intervention for community-dwelling older persons with memory problems: telemedicine versus face-to-face treatment. Int J Geriatr Psychiatry 2005;20:285-6.

13. Farabee D, Calhoun S, Veliz R. An experimental comparison of telepsychiatry and conventional psychiatry for parolees. Psychiatr Serv 2016;67:562-5.

14. O’Reilly R, Bishop J, Maddox K, Hutchinson L, Fisman M, Takhar J. Is telepsychiatry equivalent to face-toface psychiatry? Results from a randomized controlled equivalence trial. Psychiatr Serv 2007;58:836-43.

15. Kim H, Jhoo JH, Jang JW. The effect of telemedicine on cognitive decline in patients with dementia. J Telemed Telecare 2017;23:149-54.

16. Liljegren M, Naasan G, Temlett J, et al. Criminal behavior in frontotemporal dementia and Alzheimer disease. JAMA Neurol 2015;72:295-300.

17. AlDossary S, Martin-Khan MG, Bradford NK, Smith AC. A systematic review of the methodologies used to evaluate telemedicine service initiatives in hospital facilities. Int J Med Inform 2017;97:171-94. 